\title{
Childcare in Infancy and Later Obesity: a Narrative Review of Longitudinal Studies
}

\author{
Silvia Costa ${ }^{1}$ - Jean Adams ${ }^{1}$ - Sarah Gonzalez-Nahm ${ }^{2}$ - Sara E Benjamin Neelon ${ }^{1,2}$
}

Published online: 15 June 2017

(C) The Author(s) 2017. This article is an open access publication

\begin{abstract}
Purpose of Review The purpose of this review was to summarize the current literature on the longitudinal relationship between non-parental childcare during infancy and later obesity. Recent Findings Eleven studies met the inclusion criteria, comprising 74 associations relevant to the review. Studies were highly heterogeneous in terms of defining childcare, categorizing different types of childcare, assessing obesity, and age at measurement of outcome and exposure. Most of the associations were either non-significant (42 associations, $57 \%$ ) or showed a significant association between increased exposure to childcare and greater obesity (30 associations, $41 \%$ ). There were very few examples of associations indicating that childcare was associated with lower obesity.

Summary There is limited research on the longitudinal relationship between childcare in infancy and later obesity. Existing studies showed mixed results, similar to recent reviews reporting on cross-sectional studies and older ages. The different definitions of childcare and wide variety of measures of exposure make comparisons between studies challenging.
\end{abstract}

Keywords Child care $\cdot$ Nurseries $\cdot$ Infant $\cdot$ Adiposity $\cdot$ Body weight

This article is part of the Topical Collection on Obesity

Sara E Benjamin Neelon

sara.neelon@jhu.edu

1 UKCRC Centre for Diet and Activity Research (CEDAR), MRC Epidemiology Unit, School of Clinical Medicine, University of Cambridge, Cambridge, UK

2 Department of Health, Behavior and Society, Johns Hopkins Bloomberg School of Public Health, Johns Hopkins University, Baltimore, MD, USA

\section{Introduction}

High rates of childhood obesity are a worldwide concern [1]. Infancy has been repeatedly highlighted as a critical or sensitive period in post-natal life for the development and prevention of obesity [2-4]. Obesity and rapid weight gain during infancy are significantly associated with increased risk of obesity, type II diabetes, and hypertension during both childhood and adulthood [2, 4, 5]. Several individual (e.g., sex, and diet behavior), inter-personal (e.g., feeding practices), and environmental factors (e.g., attending childcare) have been reported to influence the early development of childhood obesity [6]. As environmental factors affect large numbers of children, they represent potential targets for obesity prevention at the population level [7].

A rising number of children attend out-of-home childcare starting as early as the first year of life [8], with many spending much of their week days in these settings [8,9]. Around one quarter of children up to 3 years old in developed countries attend some form of childcare [10]. Among offspring of employed mothers in the USA, an estimated $15.9 \%$ of infants aged less than 1 year and $29.8 \%$ aged one to 2 years attended an organized childcare facility (e.g., day care center or nursery) in 2011, and roughly $35 \%$ of infants received care from a grandparent [8]. The same report estimated that children aged $\leq 4$ years with employed mothers spent an average of $36 \mathrm{~h}$ per week in childcare, varying from $23 \mathrm{~h}$ with grandparents to $33 \mathrm{~h}$ in childcare centers [8]. In contrast, few infants were estimated to receive non-parental care in their own homes$4 \%$ of those aged $<1$ year and $6 \%$ of those aged $1-2$ years [8]. As such, childcare settings have become targets for intervention efforts to prevent obesity in early life [11, 12].

A number of studies have examined the relationship between childcare attendance and later obesity and found mixed results $[13 \bullet, 14 \bullet]$. The association may depend, in part, on the 
age when children first enter childcare, their total exposure, and the timing of measurement of both exposure and outcomes [13•]. Because infancy is a period of rapid physical development [2] and vulnerability [4], where children are dependent on others [15], it is crucially important to investigate childcare settings as a potential risk factor for the development of obesity. In this narrative review, we sought to examine and summarize the current evidence on the longitudinal relationship between childcare in infancy and adiposity, body mass, and obesity.

\section{Methods}

We performed a narrative review, with clear a priori definitions of participants, exposures, and outcomes of interest.

\section{Eligibility Criteria}

\section{Participants}

We included studies with children aged birth to 24 months at the time of measurement of exposure to childcare, as this is a commonly accepted age range for the infancy period [2]. This is also an increasingly recognized critical period for the development of obesity and its health consequences both in infancy and in later life $[16,17]$.

We included studies based in middle- and high-income countries; we excluded studies in low-income countries, using the World Bank's definition of a gross national income per capita of less than US\$1025 [18]. We excluded studies from these countries because of the differing economic, social and health environments in higher- versus lower-income settings, including investment in and access to health and education $[19,20]$ and leading causes of death [21]. These factors are likely to influence both the quality and use of childcare.

\section{Study Design}

We included only observational longitudinal studies, including case-control, prospective and retrospective cohort studies, and excluded all other study designs. We focused on observational designs to allow the exploration of associations between childcare and the outcomes of interest as they exist in the general population and in real-life settings, rather than in experimental situations and settings. Including only longitudinal studies helped reduce the possibility of reverse causality between receipt of childcare and obesity.

\section{Exposure and Comparator}

The exposure of interest was non-parental childcare, versus a comparator of parental care. If the exposure was measured as a categorical variable (e.g., parental care and formal nonparental childcare), the comparator of interest was parental care. In some cases authors used the term "no childcare" and we assumed this to be parental care. If the exposure was measured as a continuous variable (e.g., number of hours of nonparental childcare per week), we extracted change in outcome per unit increase in change in exposure. Where possible, we did not impose any standard definitions of specific types of childcare and instead used the authors' language. In one case, after discussion with the author, we grouped all care provided in someone else's or the child's own home as "informal care" irrespective of who provided it [9].

\section{Outcomes}

The outcomes of interest included adiposity or body mass ("obesity") directly measured by researchers, childcare staff or parents, or overweight or obesity, at a time point subsequent to recording of childcare use. Examples of accepted measures included weight, body mass index (BMI), waist or hip circumference, weight-for-length (WFL) ratio, skinfold thickness, fat mass, and overweight or obesity status. We excluded studies using self-reported measures.

\section{Analyses}

We only included studies that used multivariable analysis to account for potential confounders, such as maternal smoking during pregnancy, birth weight, duration of breastfeeding, and socioeconomic status. This decision was based on existing evidence that these variables are associated with obesity in both childhood and adulthood [4, 5] and may be associated with the decision to use non-parental childcare and so have the potential to confound any relationship between childcare and obesity.

\section{Literature Search and Synthesis}

One author (SC) conducted the literature search using a threestage process. Firstly, she scanned the bibliography of recent literature reviews $[13 \bullet, 14 \bullet]$ covering the relationship between childcare in preschool age children and overweight and obesity. From these, she identified potentially relevant studies for which full texts were retrieved and screened against the eligibility criteria. Next, she scanned the bibliographies of included studies, identified potentially relevant studies, and retrieved and screened full texts against the eligibility criteria. There were no searches of publication databases. We included both papers published in peer-reviewed journals and published theses. Theses are generally considered by two examiners and revised accordingly - a process arguably similar to peer review of journal articles. Finally, we searched the Google Scholar for citations of included studies identified in the first 
two steps. We then identified potentially relevant studies and retrieved and screened full texts against the eligibility criteria. We then tabulated key study information and performed a narrative synthesis of results, exploring patterns according to type of childcare, timing of measurement of childcare exposure, timing of measurement of obesity-related outcomes, and country of origin.

\section{Results}

\section{Summary of Included Studies}

We included 11 studies - two thesis [22, 23] and nine peerreviewed articles [9, 24-31] - that met the eligibility criteria. All studies were published within the last 10 years (20082016) and were based in high-income countries [18], with over one third of the studies $(n=4)$ originating from the USA (Table 1). Sample sizes varied widely (range 105 to 27,821 participants), with no studies restricted by child sex, race or ethnicity. All studies reported on more than one relevant association, resulting from using more than one measure of either exposure, outcome or both; measuring exposure, outcome or both at more than one time point; and sub-group analyses. Therefore, although only 11 studies met the inclusion criteria, we included 74 relevant associations. As exposure and outcome measures were very heterogeneous between and within studies, with sometimes multiple exposure and outcome measures in the same study, we report our results at the level of individual associations rather than at the studylevel for the remainder of the review. We believe that this helps both to demonstrate the heterogeneity in the literature, detail the full extent of knowledge in this area, and make progress towards exploring sources of heterogeneity in reported results.

The most common exposure variable was duration of childcare exposure ( $n=26$ associations, 35\%) [9, 24, 27, 31 , measured as either time per week or years, or categorized as full- versus part-time attendance. The next most frequent was a binary measure of any childcare use or not $(n=21$ associations, 28\%) [22, 25, 26, 29], where parental care was the main comparator. Age at childcare exposure was 012 months for $82 \%$ of the associations studied [9, 22, 24, 25, 27-30]. The most common outcomes were weight gain ( $n=24$ associations, $32 \%$-although weight gain was only included as an outcome in one study) [27]; overweight or obese status ( $n=19$ associations, 26\%) [23-25, 28-30]; and BMI $z$ score $(n=19$ associations, 26\%) [9, 24-26, 29]. None of the studies included crossing weight percentiles as outcomes. Both overweight or obese status and BMI $z$ score were calculated using a variety of different growth references and standards (Table 2). Outcome variables were measured at 0 12 months in $42(57 \%)$ of the associations studied [9, 22, 24,
$25,27]$, with 11 years [29] being the oldest age at outcome measurement.

Potential confounders included in multivariable analyses were relatively consistent among studies, and included indicators of socioeconomic status (e.g., household income and parental education level), maternal characteristics (e.g., age, pre-pregnancy BMI, and smoking during pregnancy), and child's characteristics and early life factors (e.g., gender, birth weight, and breastfeeding duration). However, only four studies $[24,26,28,30]$ explicitly attempted to investigate the potential mediating role of some of these factors, such as breastfeeding duration. Results were highly heterogeneous. For example, some studies reported positive associations between attending childcare and risk of later overweight or obesity $[24,25]$ or increased adiposity indicators [24-27], while others reported negative [22] or no association [23, 27, 30].

\section{Synthesis of Findings}

\section{Exposure to Any Versus No Childcare}

Seven studies included analyses of any non-parental childcare (undifferentiated further by authors) versus parental childcare $[9,22,24-26,29,31]$. Out of 29 relevant associations 14 $(48 \%)$ were not significant, 11 (38\%) showed significant positive associations, and four (14\%) showed significant negative associations. Considering the method of exposure measurement (e.g., continuous versus binary), or outcome measurement (e.g., overweight status or BMI $z$ score) did not reveal any particular patterns.

\section{Exposure to Specific Types of Childcare}

Associations between exposure to informal childcare and adiposity outcomes were assessed in five studies [9, 23, 24, 27, $30]$. Out of 15 associations, five (33\%) were significant and positive. The remaining ten $(67 \%)$ were not significant. No discernible patterns were seen in relation to type of outcome measured. Five studies included assessment of the association between exposure to formal childcare and outcomes of interest. Of 12 relevant associations, the majority $(n=9,75 \%)$ were non-significant, and only three $(25 \%)$ showed a positive and significant relationship. Perhaps of note, all of these significant associations were reported in the same study [24].

\section{Age at Measurement of Childcare Exposure}

Exposure to childcare was measured at $<12$ months in the majority of associations $(n=57,77 \%)$. Of these $35(61 \%)$ were not significant, $18(32 \%)$ associations were significant and positive, and four (7\%) showed a significant and negative effect. Of the 17 associations that included a measurement of childcare exposure at $\geq 12$ months, $12(70 \%)$ were not 


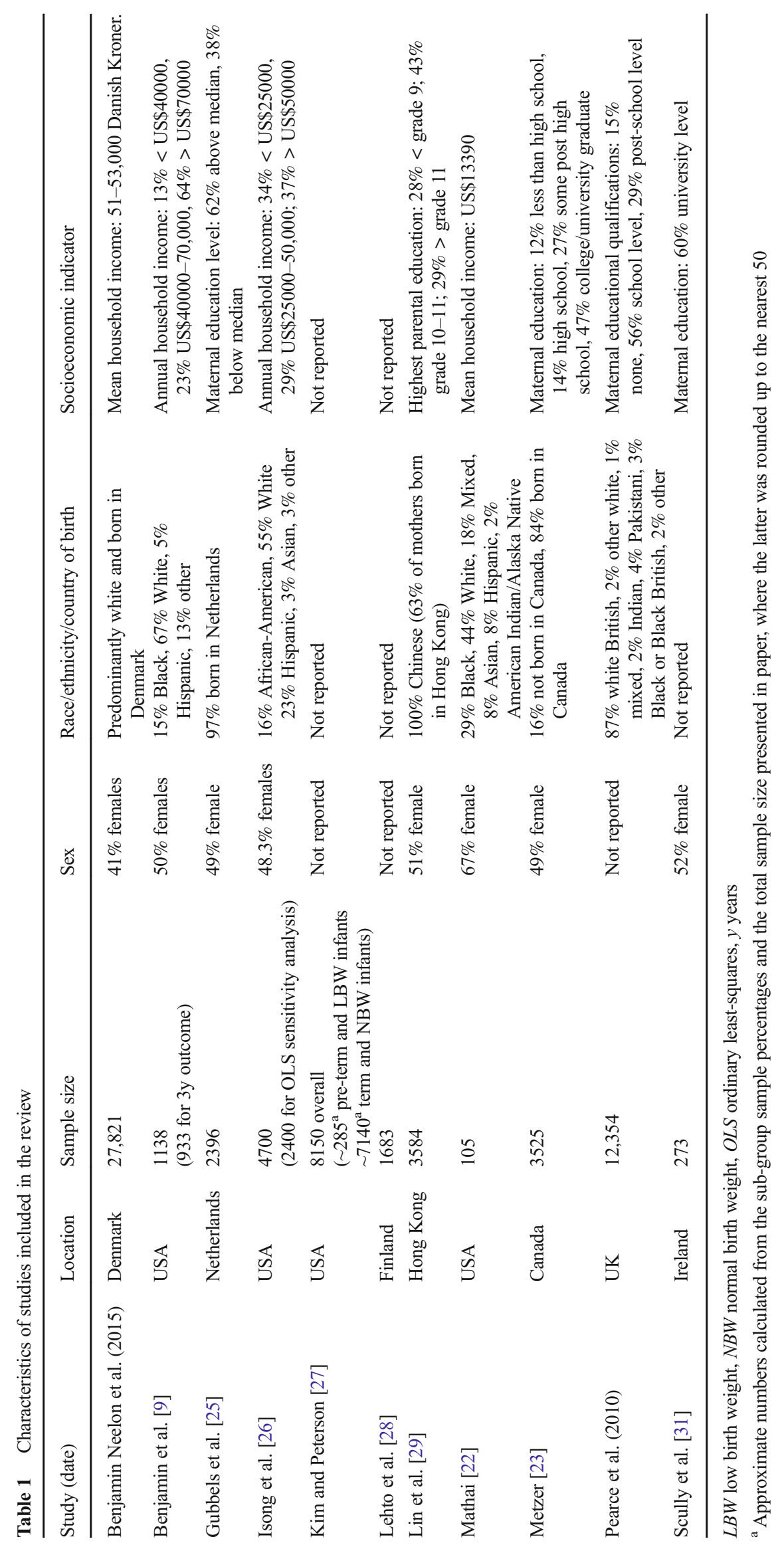




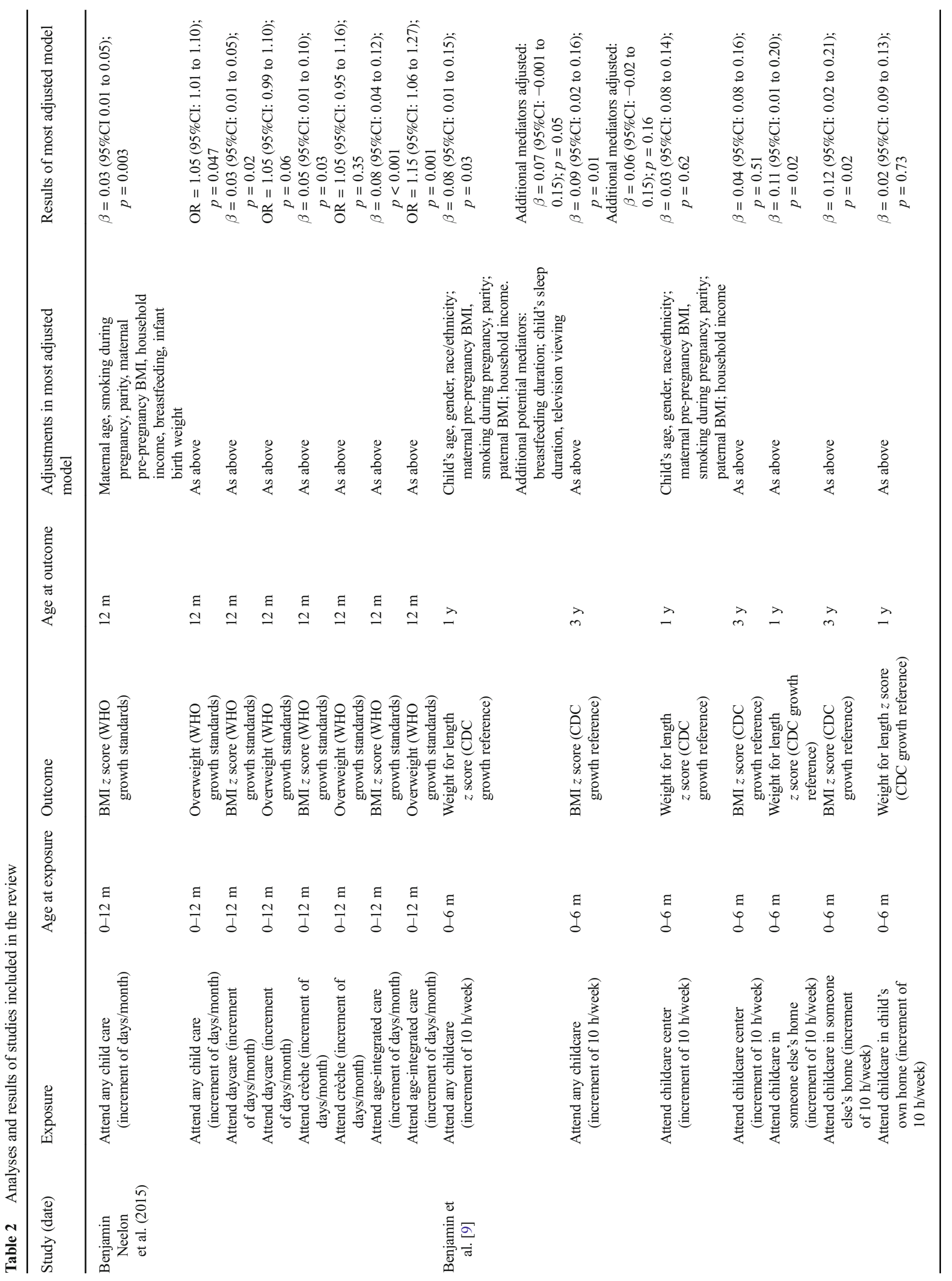




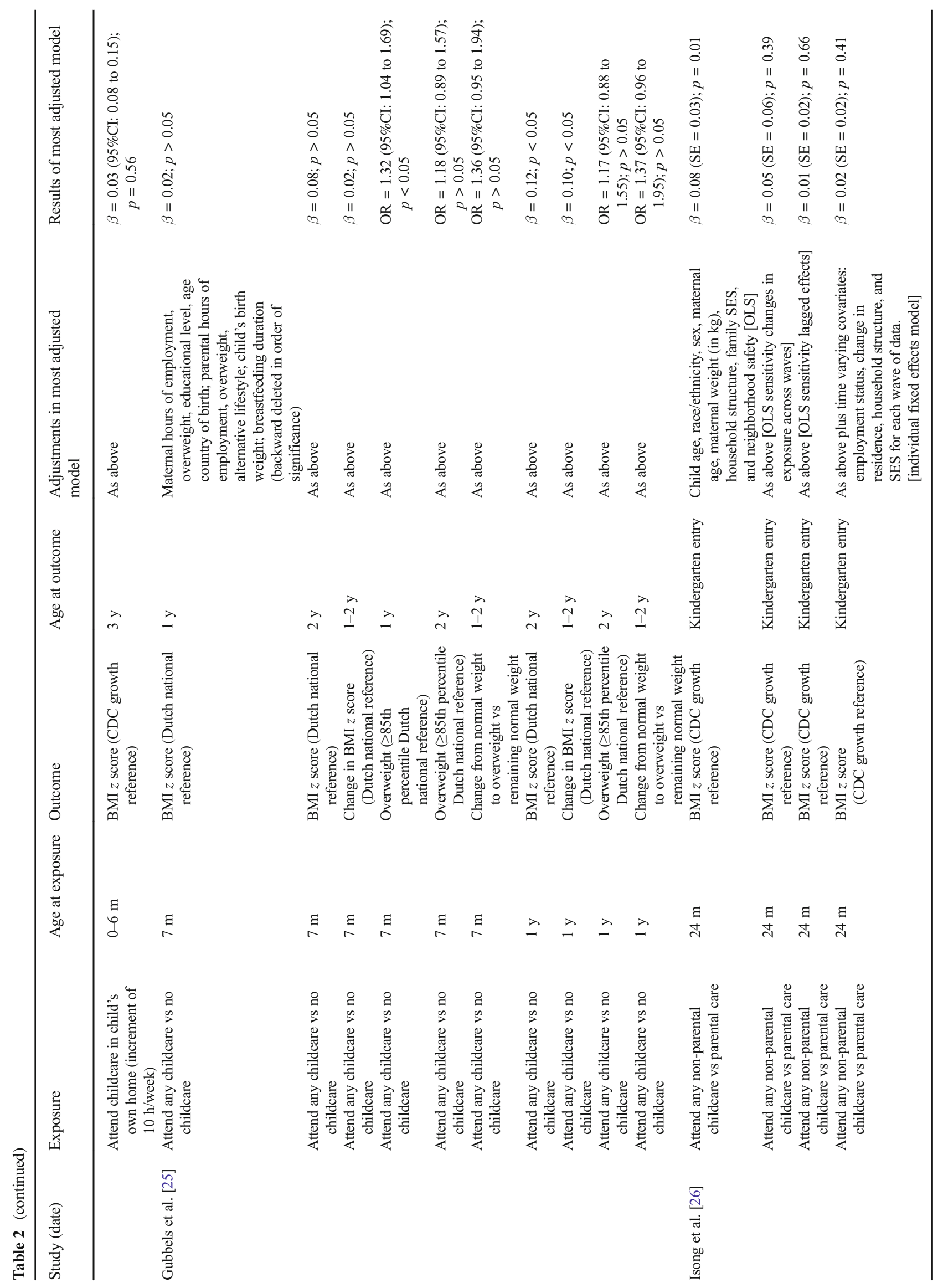




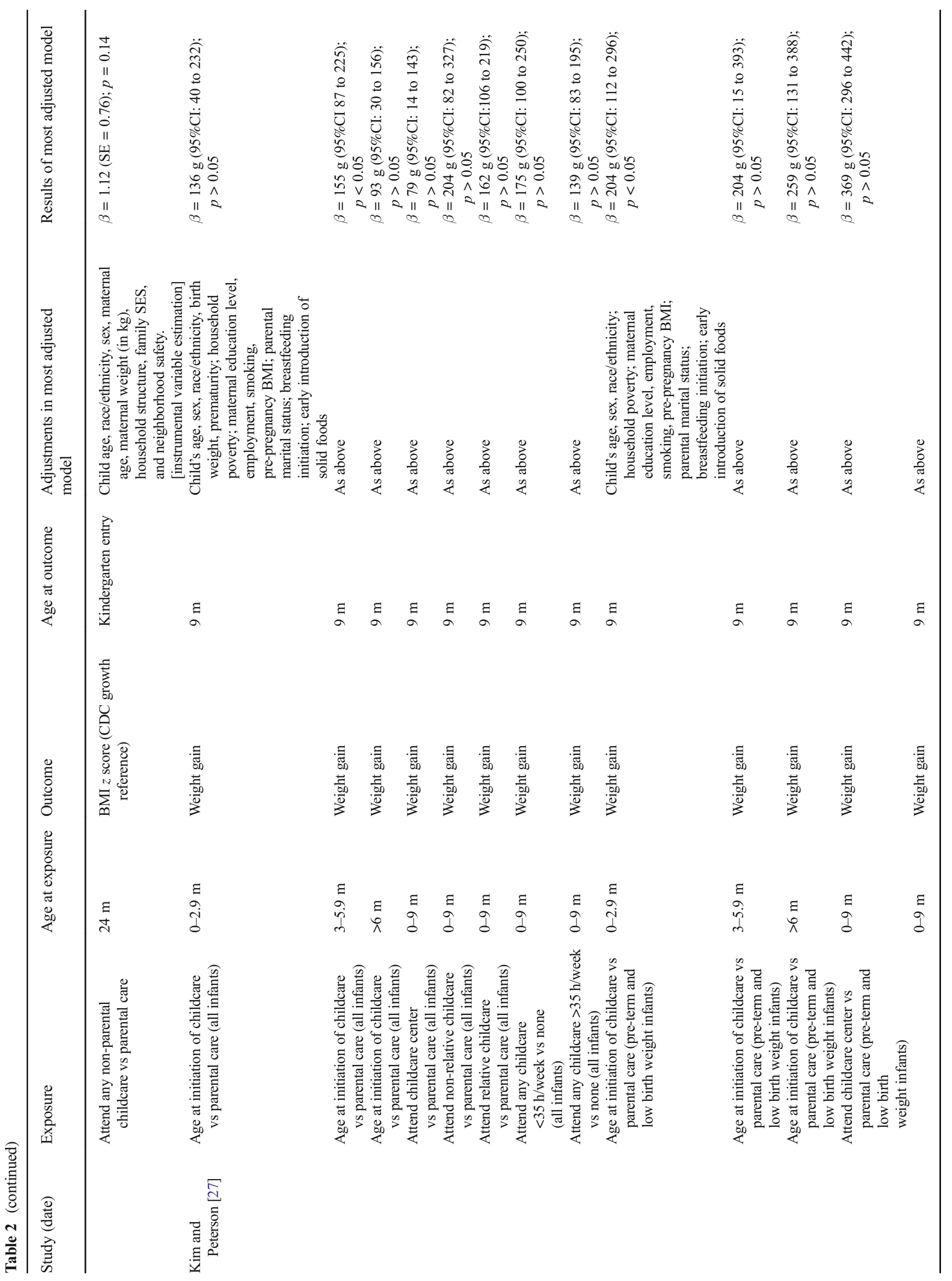




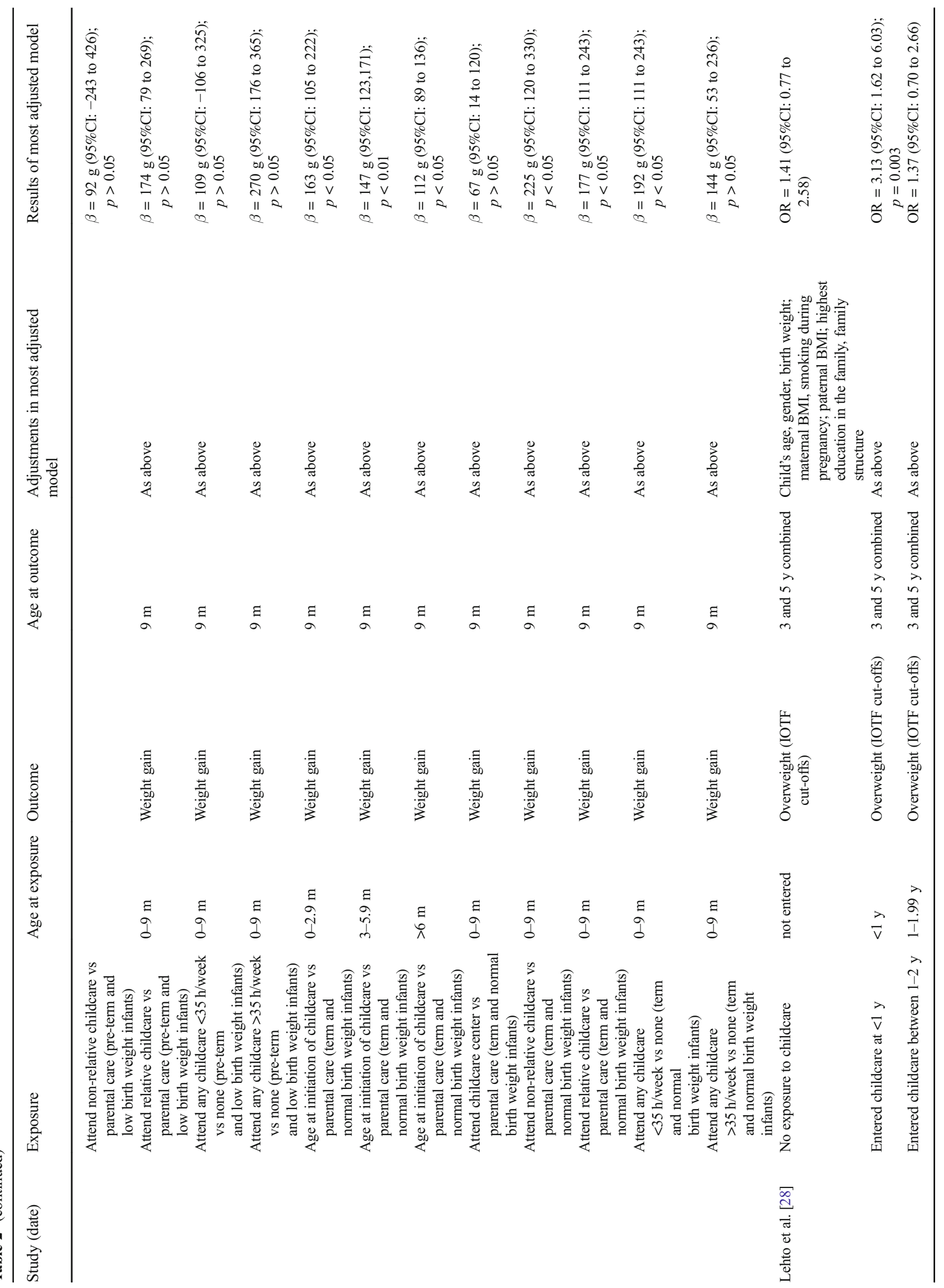




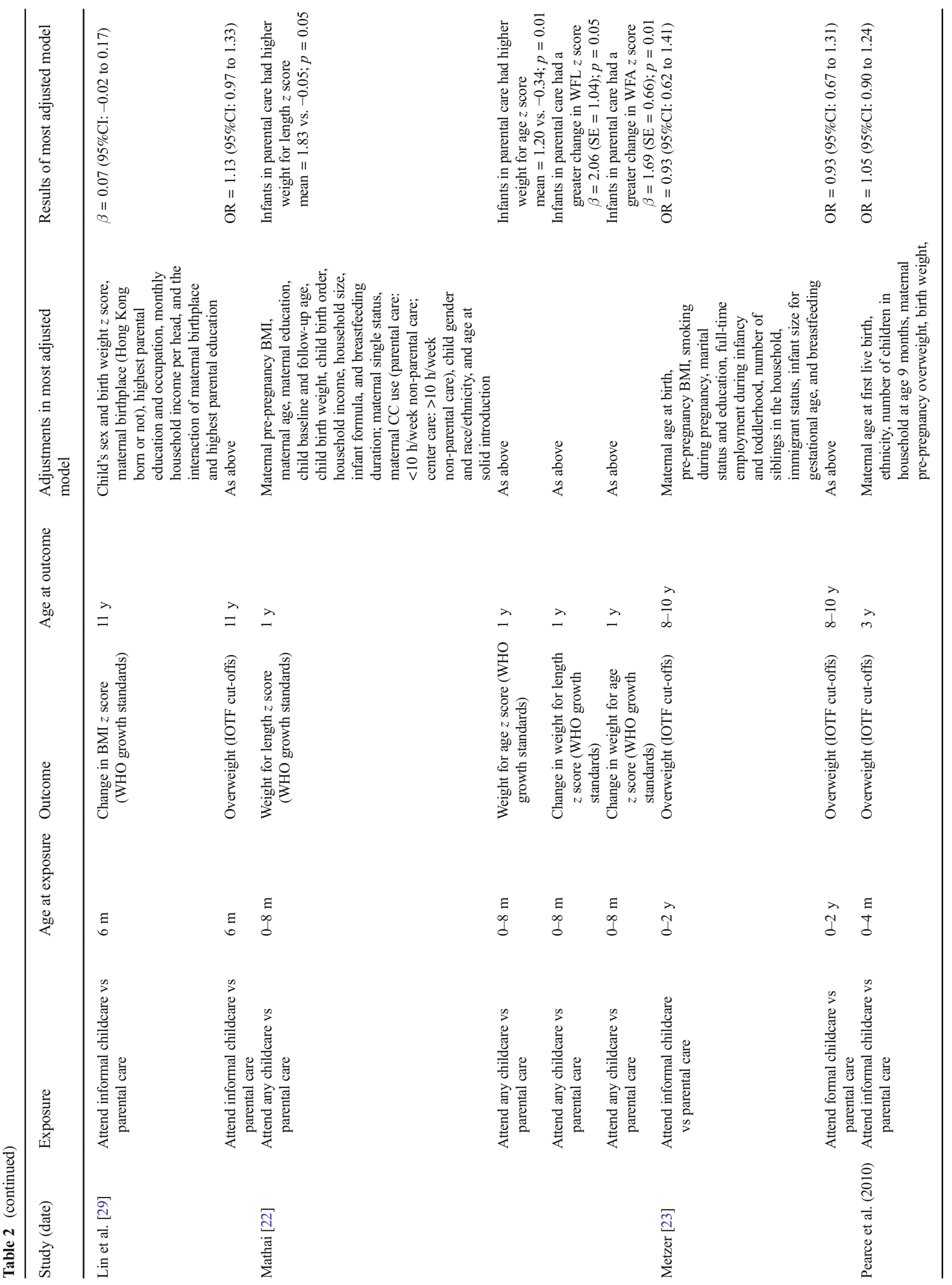




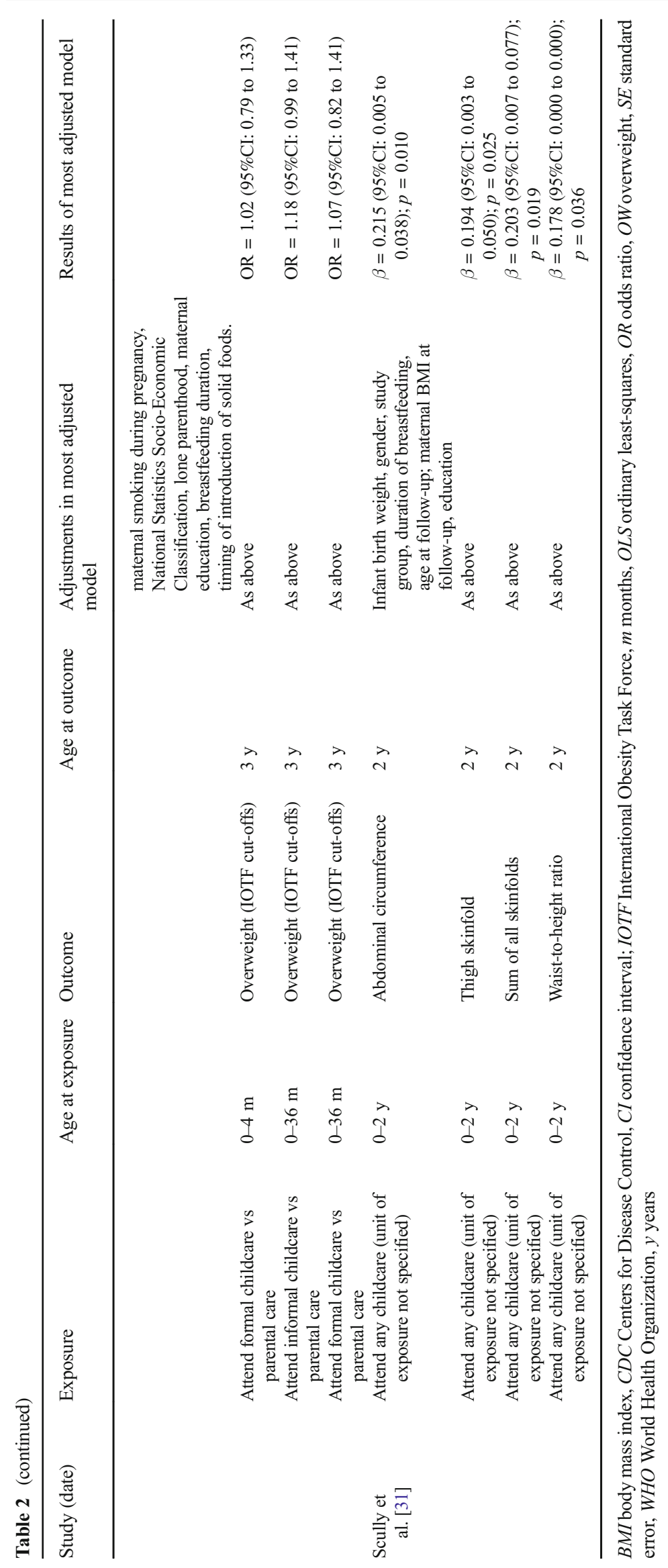


significant, with the remaining five (30\%) showing significant positive associations.

\section{Age at Measurement of Outcome}

Age at measurement of outcome was 0-24 months in most studies ( $n=54$ associations, $73 \%)$. Of these, $28(52 \%)$ associations were not significant, $22(41 \%)$ were significant and positive, and the remaining four (7\%) were significant and negative. In the $16(22 \%)$ associations where age of measurement was 25-71 months, 11 (69\%) were not significant and five $(31 \%)$ were significant positive associations. No significant associations were observed between childcare and overweight or adiposity indicators measured at $\geq 72$ months (6 years), although such long-term follow-up was only included in four $(5 \%)$ associations from two studies [23, 29].

\section{Country of Origin}

In studies originating from the USA (four studies; 41 associations) $[9,22,26,27]$, most associations were non-significant $(n=26,63 \%)$. However, in 11 cases $(27 \%)$ there was a significant positive association, and the remaining four (11\%) reported a significant negative association. In studies from countries other than the USA (seven studies; 33 associations) [23-25, 28-31], 18 (55\%) associations were non-significant, and $15(45 \%)$ were significant and positive.

\section{Discussion}

We used reproducible methods to conduct a narrative review of the longitudinal association between childcare exposure during infancy and later adiposity. We found 11 studies that met our inclusion criteria, reporting a total of 74 relevant associations. Overall, the majority of associations were nonsignificant or showed positive associations indicating that increased exposure to childcare was associated with greater obesity. There were very few examples of significant negative associations, where increased childcare exposure was associated with lower obesity or adiposity indicators, and these were all from the same study [22]. The authors propose that the food provided in childcare (more processed foods) compared to parental care (more in line with infant feeding guidelines) may, paradoxically, explain this difference. However, it is also possible that these "inconsistent" findings merely reflect expected variation. Studies were highly heterogeneous in terms of defining childcare, categorizing different types of childcare, assessing obesity and adiposity, and age at measurement of outcome and exposure. Although we attempted to group studies and associations meaningfully (e.g., by type of exposure), this did not reveal any clear patterns.
One striking finding from the review is the heterogeneity of the exposures studied. While some authors compared any nonparental care to parental care, others focus on particular types of care, seeking to differentiate between, for example, informal and formal care, and different sub-categories within these. Furthermore, the definitions of different types of childcare were not consistent. For example, different authors use "informal childcare" to mean both unlicensed but paid-for care, grandparental care, and some center-based care. These differences in how the exposure was defined undoubtedly contributed to some of the heterogeneity in findings. Development of a clear typology of childcare and definitions of different types of care, alongside clear reporting of what types of care are considered in different studies would likely add some clarity to the current literature. However, international differences in types of childcare available may complicate this process.

The heterogeneity of findings in this review suggests that any effect of childcare in infancy on the development of obesity may not be large, although even a small effect at the individual level may have significant implications at the population level. Specific factors related to the quality and context of the childcare environment may be important. The nutritional and physical activity environments provided by different types of childcare, and by different providers within broad types, can vary greatly [32-34]. Although all included studies controlled for a variety of known confounders, none considered the potential mediating or moderating roles of childcare-specific factors such as dietary provisions (other than breastfeeding), sleep, or physical activity opportunities. Thus, the variability in childcare environments may partly explain the heterogeneity of study findings.

It is also possible that the effects of childcare on obesity vary according to child and family characteristics, such as socioeconomic position. There is expanding evidence that some public health interventions are more effective in affluent groups [35] and that some risk factors are more potent in less affluent groups [36]. Again, such effects may obscure findings at the wider group level, such as those studied here. Further consideration of child- and family-level variables as effect modifiers, rather than just confounders may yield valuable insights.

In addition to variations in effect according to family characteristics, use of some types of childcare may be restricted to particular types of families. In these cases, lack of variability in family characteristics within childcare types may mean that statistical adjustment for family characteristics is inadequate and that uncontrolled confounding by family characteristics persists. For example, in five studies looking at the use of formal childcare, only one [24] found positive associations with overweight or BMI $z$ score. The latter was set in Denmark, where paid parental leave can be taken for up to 52 weeks and shared by both parents. Danish parents using crèches or age-integrated facilities in the first year of their child's life may, therefore, be a select group with particular characteristics (e.g., ineligible for paid parental leave because 
they are recent immigrants). These characteristics may themselves influence the development of obesity and so act as confounders in any relationship between childcare and obesity [37]. Thus, the reported significant positive associations between formal childcare and both overweight and BMI $z$ score may be a reflection of uncontrolled confounding, rather than a reflection of the influence of this type of childcare in the development of obesity.

We did not find strong evidence that exposure to childcare in the first year of life was any more or less harmful than exposure in the second year. However, given the variety of ways in which exposure was assessed, and the number of different outcome measures used, it is difficult to draw clear conclusions. It is possible that younger children are no more vulnerable to the effects of childcare than older children. However, we did not find any studies that tested this hypothesis directly and further work is required to assess whether any effects of childcare on obesity vary according to the timing of exposure.

We did find some indication that the effects of childcare in infancy on obesity may diminish over time. While only four associations in included studies included follow-up of children beyond 6 years, none of these were statistically significant. Thus, it may be possible that any harmful effects of childcare in infancy are not sustained into later childhood or beyond. Further longitudinal studies, with follow-up into middle childhood and beyond, are required to assess any effects of early childcare on later obesity.

One potential mechanism linking childcare and obesity is the early discontinuation of breastfeeding. Exclusive breastfeeding and breastfeeding duration of a minimum of four to 6 months have been consistently linked to a lower likelihood of obesity in childhood [38-40]. Research has shown that women who return to work within the first 4 months postpartum and need to rely on out-of-home childcare are less likely to continue breastfeeding [41-44]. Two papers included in this review found that breastfeeding did not mediate the association between childcare and obesity [23, 30]; however, neither of these examined exclusive breastfeeding. The timing of mothers' return to work is influenced, in part, by the amount of maternity leave available to mothers - which varies globally. While adequate maternity leave policies may help encourage women to breastfeed their infants, they are only one of multiple factors affecting a woman's ability and decision to exclusively breastfeed. Childcare policies that support continued breastfeeding [45] may help women continue to exclusively breastfeed their infants in childcare, which may result in lower rates of child obesity over time.

\section{Limitations}

While we used reproducible methods to find relevant papers for inclusion in this review, we did not conduct a systematic review. In particular, we did not conduct an exhaustive search of relevant databases such as MEDLINE, CINAHL or Embase. We may not, therefore, have included all relevant studies meeting the eligibility criteria. Furthermore, by including published theses, we may have incorporated some less rigorously assessed material than may be found in peer-reviewed journal articles. However, these theses make an important contribution to what is currently a very small volume of relevant literature in this area. In addition, we did not perform all aspects of searching and screening in duplicate which may have led to additional errors. We are currently conducting a systematic review examining the association of childcare factors with diet, physical activity, sedentary behavior, sleep, and stress that will help overcome these limitations [46]. We also restricted the review to longitudinal data in order to reduce the possibility of reverse causality [47]. However, longitudinal studies alone cannot determine causality [48] and we did not conduct a formal quality assessment of included studies. While it would likely be very difficult to gain consent and parental agreement to conduct a randomized controlled trial of, e.g., parental versus non-parental care, a variety of more innovative methods could be used to study the effects of childcare on obesity —including quasi-experimental evaluations of changes in national and local childcare and parental leave policies. The studies included in our review were also all conducted in high-income countries, and one third were from the USA alone. Thus, our findings may not be generalizable to other countries not represented, particularly middle-income countries.

\section{Conclusions}

Although our results were heterogeneous, they suggest the association between childcare and obesity is likely to be neutral or harmful in terms of later adiposity and obesity. As such, there is a clear need for further research on this topic. Longitudinal research is resource intensive and researchers must often rely on existing cohort studies, with questions and variables, which were not guided by any specific research questions at the outset. While we recognize this limitation on what can be studied, it does not diminish the need to ensure that future studies are informed by theory. Further studies should also seek to include longer follow-up periods to determine whether, how, for whom, and under what circumstances any harmful effects of childcare on obesity persist into later childhood and beyond.

Although we cannot conclude from the available evidence that childcare is definitely a risk factor for obesity, we found almost no evidence that it is protective. Until it is clarified whether childcare has a neutral or harmful effect on obesity, it is important to ensure that all childcare settings promote the health of the children in childcare, particularly in terms of the quality and quantity of foods offered and opportunities for physical activity. 
Overall, we found few longitudinal studies exploring the association between childcare in infancy with later obesity. Further research is required to develop a typology of childcare and stronger conceptual theory about how childcare may influence the development of obesity. These theories could be used to drive future testing of pre-specified hypotheses concerning when, how, for whom and under what circumstances childcare in infancy may lead to obesity in later life. Studies with longer follow-up into later childhood and beyond are also required to determine any long-term public health impact of childcare. The available evidence is neither comprehensive nor consistent enough to warrant concern about infants using childcare. However, given the increasing numbers of children attending childcare and the amount of time children spend in these settings, it is sensible to ensure childcare environments promote the health of all children.

Acknowledgements The work was undertaken by the Centre for Diet and Activity Research (CEDAR), a UKCRC Public Health Research Centre of Excellence. Funding from the British Heart Foundation, Cancer Research UK, Economic and Social Research Council, Medical Research Council, the National Institute for Health Research, and the Wellcome Trust, under the auspices of the UK Clinical Research Collaboration, is gratefully acknowledged.

\section{Compliance with Ethical Standards}

Conflict of Interest Silvia Costa, Jean Adams, Sarah Gonzalez-Nahm, and Sara E Benjamin Neelon each declare no potential conflicts of interest.

Human and Animal Rights and Informed Consent This article does not contain any studies with human or animal subjects performed by any of the authors.

Open Access This article is distributed under the terms of the Creative Commons Attribution 4.0 International License (http:// creativecommons.org/licenses/by/4.0/), which permits unrestricted use, distribution, and reproduction in any medium, provided you give appropriate credit to the original author(s) and the source, provide a link to the Creative Commons license, and indicate if changes were made.

\section{References}

Papers of particular interest, published recently, have been highlighted as:

- Of importance

1. World Health Organization. Global status report on noncommunicable diseases 2014. Geneva: World Health Organization; 2014.

2. Cameron N, Demerath EW. Critical periods in human growth and their relationship to diseases of aging. Am J Phys Anthropol. 2002;119 doi:10.1002/ajpa.10183.

3. Dietz WH. Critical periods in childhood for the development of obesity. Am J Clin Nutr. 1994;59

4. Lakshman R, Elks CE, Ong KK. Childhood obesity. Circulation. 2012;126:1770-9. doi:10.1161/CIRCULATIONAHA.111.047738.
5. Brisbois TD, Farmer AP, McCargar LJ. Early markers of adult obesity: a review. Obes Rev. 2012;13 doi:10.1111/j.1467-789X. 2011.00965.x.

6. Davison KK, Birch LL. Childhood overweight: a contextual model and recommendations for future research. Obes Rev. 2001;2 doi:10. 1046/j.1467-789x.2001.00036.x.

7. Leal C, Chaix B. The influence of geographic life environments on cardiometabolic risk factors: a systematic review, a methodological assessment and a research agenda. Obes Rev. 2011;12(3):217-30. doi:10.1111/j.1467-789X.2010.00726.x.

8. Laughlin L. Who's minding the kids? Child care arrangements: Spring 2011. Washington, DC: Bureau C 2011; 2013.

9. Benjamin SE, Rifas-Shiman SL, Taveras EM, et al. Early child care and adiposity at ages 1 and 3 years. Pediatrics. 2009;124 doi:10. 1542/peds.2008-2857.

10. United Nations Children's Fund (UNICEF). The Childcare Transition Innocenti Report Card. Florence, 2008.

11. Zhou YE, Emerson JS, Levine RS, Kihlberg CJ, Hull PC. Childhood obesity prevention interventions in childcare settings: systematic review of randomized and nonrandomized controlled trials. Am J Health Promot. 2013;28 doi:10.4278/ajhp.121129LIT-579.

12. Institute of Medicine. Early childhood obesity prevention policies. Washington DC, 2011.

13. Swyden K, Sisson SB, Lora K, Castle S, Copeland KA. Association of childcare arrangement with overweight and obesity in preschoolaged children: a narrative review of literature. Int $\mathrm{J}$ Obes. 2017;41(1):1-12. doi:10.1038/ijo.2016.198. Important recent narrative review investigating the same topic in preschool children, where some information for infants exists

14. Alberdi G, McNamara AE, Lindsay KL, et al. The association between childcare and risk of childhood overweight and obesity in children aged 5 years and under: a systematic review. Eur J Pediatr. 2016;175(10):1277-94. doi:10.1007/s00431-016-2768-9. Important recent systematic review investigating the same topic in children aged $\leq 5$ years, where some information for infants exists

15. Bogin B. Patterns of human growth. 2nd edition ed. Cambridge: Cambridge University Press; 1999.

16. Woo Baidal JA, Locks LM, Cheng ER, Blake-Lamb TL, Perkins ME, Taveras EM. Risk factors for childhood obesity in the first 1, 000 days: a systematic review. Am J Prev Med. 2016;50(6):761-79. doi:10.1016/j.amepre.2015.11.012.

17. Black RE, Victora CG, Walker SP, et al. Maternal and child undernutrition and overweight in low-income and middle-income countries. Lancet. 2013;382(9890):427-51. doi:10.1016/S01406736(13)60937-X.

18. The World Bank. World Bank Country and Lending Groups, World Bank Data Help Desk. Washington, DC, USA. 2017. https:// datahelpdesk.worldbank.org/knowledgebase/articles/906519world-bank-country-and-lending-groups. Accessed 18/01/2017.

19. Orach CG. Health equity: challenges in low income countries. Afr Health Sci. 2009;9

20. Fürnkranz-Prskawetz A, Lee RD, Lee S-H et al. Lower-income countries and the demographic dividend. Honolulu, 2012.

21. World Health Organization. The top 10 causes of death. In: Fact sheet. Geneva: World Health Organization; 2012.

22. Mathai RA. Comparison of infant feeding practices, nutrient intake and body weights by childcare use. Urbana, Illinois: University of Illinois at Urbana-Champaign; 2012.

23. Metzer NV. Maternal employment during infancy and toddlerhood: mechanisms for associations with childhood overweight/obesity in Canada. Ontario: The University of Western Ontario; 2014.

24. Benjamin Neelon SE, Schou Andersen C, Schmidt Morgen C, et al. Early child care and obesity at 12 months of age in the Danish 
National Birth Cohort. Int J Obes. 2015a;39(1):33-8. doi:10.1038/ ijo.2014.173.

25. Gubbels JS, Kremers SP, Stafleu A, et al. Child-care use and the association with body mass index and overweight in children from 7 months to 2 years of age. Int J Obes. 2010;34(10):1480-6. doi:10. 1038/ijo.2010.100.

26. Isong IA, Richmond T, Kawachi I, Avendaño M. Childcare attendance and obesity risk. Pediatrics. 2016;138(5):e20161539. doi:10. 1542/peds.2016-1539.

27. Kim J, Peterson KE. Association of infant child care with infant feeding practices and weight gain among US infants. Archives of Pediatrics \& Adolescent Medicine. 2008;162 doi:10.1001/archpedi. 162.7.627.

28. Lehto R, Mäki P, Ray C, Laatikainen T, Roos E. Childcare use and overweight in Finland: cross-sectional and retrospective associations among 3- and 5-year-old children. Pediatric Obesity. 2016;11(2):136-43. doi:10.1111/ijpo.12036.

29. Lin SL, Leung GM, Hui LL, Lam TH, Schooling CM. Is informal child care associated with childhood obesity? Evidence from Hong Kong's "children of 1997" birth cohort. Int J Epidemiol. 2011;40 doi:10.1093/ije/dyr086.

30. Pearce A, Li L, Abbas J, Ferguson B, Graham H, Law C. Is childcare associated with the risk of overweight and obesity in the early years? Findings from the UK Millennium Cohort Study. Int J Obes. 2005;2010:34. doi:10.1038/ijo.2010.15.

31. Scully H, Alberdi G, Segurado R, et al. Child care exposure influences childhood adiposity at 2 years: analysis from the ROLO Study. Childhood Obesity (Print). 2016; doi:10.1089/chi.2016. 0127.

32. Benjamin Neelon SE, Vaughn A, Ball SC, McWilliams C, Ward DS. Nutrition practices and mealtime environments of North Carolina child care centers. Childhood Obesity (Print). 2012;8(3): 216-23. doi:10.1089/chi.2011.0065.

33. Gubbels JS, Van Kann DHH, Jansen MWJ. Play equipment, physical activity opportunities, and Children's activity levels at childcare. J Environ Public Health. 2012;2012:8. doi:10.1155/ 2012/326520.

34. Kaphingst KM, Story M. Child care as an untapped setting for obesity prevention: state child care licensing regulations related to nutrition, physical activity, and media use for preschool-aged children in the United States. Prev Chronic Dis. 2009;6(1):A11.

35. Adams J, Mytton O, White M, Monsivais P. Why are some population interventions for diet and obesity more equitable and effective than others? The role of individual agency. PLoS Med. 2016;13(4): e1001990. doi:10.1371/journal.pmed.1001990.

36. Macintyre S. Deprivation amplification revisited; or, is it always true that poorer places have poorer access to resources for healthy diets and physical activity? Int J Behav Nutr Phys Act. 2007;4(1): 32. doi:10.1186/1479-5868-4-32.
37. Gadd M, Sundquist J, Johansson S-E, Wändell P. Do immigrants have an increased prevalence of unhealthy behaviours and risk factors for coronary heart disease? Eur J Cardiovasc Prev Rehabil. 2005;12(6):535-41. doi:10.1097/01.hjr.0000174829.25388.ed.

38. Gibson LA, Hernandez Alava M, Kelly MP, Campbell MJ. The effects of breastfeeding on childhood BMI: a propensity score matching approach. Journal of Public Health (Oxford, England). 2016; doi:10.1093/pubmed/fdw093.

39. Lefebvre CM, John RM. The effect of breastfeeding on childhood overweight and obesity: a systematic review of the literature. Journal of the American Association of Nurse Practitioners. 2014;26(7):386-401. doi:10.1002/2327-6924.12036.

40. Yeung H, Leff M, Rhee KE. Effect of exclusive breastfeeding among overweight and obese mothers on infant weight-for-length percentile at 1 year. Breastfeeding Medicine : the official journal of the Academy of Breastfeeding Medicine. 2017;12:39-47. doi:10. 1089/bfm.2016.0071.

41. Bai DL, Fong DY, Tarrant M. Factors associated with breastfeeding duration and exclusivity in mothers returning to paid employment postpartum. Matern Child Health J. 2015;19(5):990-9. doi:10. 1007/s10995-014-1596-7.

42. Kim J, Gallien TL. Childcare arrangements and infant feeding practices by family structure and household income among US children aged 0 to 2 years. Maternal \& Child Nutrition. 2016;12(3):591602. doi:10.1111/mcn.12152.

43. Pearce A, Li L, Abbas J, et al. Childcare use and inequalities in breastfeeding: findings from the UK Millennium Cohort Study. Arch Dis Child. 2012;97(1):39-42. doi:10.1136/adc.2009.177337.

44. Shim JE, Kim J, Heiniger JB. Breastfeeding duration in relation to child care arrangement and participation in the special supplemental nutrition program for women, infants, and children. Journal of Human Lactation : official journal of International Lactation Consultant Association. 2012;28(1):28-35. doi:10.1177/ 0890334411424728.

45. Benjamin Neelon SE, Duncan DT, Burgoine T, Mayhew M, Platt A. Promoting breastfeeding in child care through state regulation. Matern Child Health J. 2015b;19(4):745-54. doi:10.1007/s10995014-1560-6.

46. Costa S, Adams J, Phillips V, Benjamin Neelon SE. The relationship between childcare and adiposity, body mass and obesityrelated risk factors: protocol for a systematic review of longitudinal studies. Systematic Reviews. 2016;5(1):141. doi:10.1186/s13643016-0312-7.

47. Rajulton F. The fundamentals of longitudinal research: an overview. Canadian Studies in Population. 2001;28

48. Hill AB. The environment and disease: association or causation? Proceedings of the Royal Society of Medicine. 1965;58(5):295300. 